\title{
Vascular Dysfunction in Aging and Related Disease
}

\author{
Dr. Pranjali Deshpande ${ }^{1}$, Dr. Deepak S. Howale ${ }^{* 2}$ \\ ${ }^{1}$ Associate Professor Dept. of Physiology Zydus Medical College and Hospital, Dahod \\ ${ }^{2}$ Dean Govt. Medical College, Silvassa
}

Address for Correspondence

Dr. Deepak S. Howale

Dean Govt. Medical College, Silvassa

\begin{abstract}
The process that importantly impact on their health status of elder people represents vascular aging. Aging process is one of the factors for main risk for the development of cardiovascular disease (CVD). Many studies show that 50\% clinical CVD present in the elderly. Many studies epidemiologically show that risk factor for CVD is age. Actually the prevalence and incidence of coronary heart disease, hypertension, congestive heart failure and stroke steeply increase with age. Individuals in 75-84 year old as compared to 35-44 year old people increase in death rates for heart disease and cerebrovascular disease there is a $\sim 60$ fold and $\sim 0$ fold respectively. The endothelium plays an active role in process of platelet adhesion and inflammation. C-reactive protein (CRP) is one of the acute phase proteins. The aim of study was screening of population with asymptomatic individuals for risk markers of hypertension and atherosclerosis leading to vascular events and also investigation of recently detected hypertension and diabetes mellitus. Materials and methods: This study was carried out in Zydus Medical College and hospital, Dahod. In this study total 200 indivuals were included. 2 control groups were include as 38 Group I healthy controls (Age range: 18-24 years) and 65 Group II controls included apparently healthy, asymptomatic individuals whose blood pressure values did not exceed 139/89 and whose fasting blood sugar (FBS) values were B110 mg\% (Age range: 25-60 years). It is also grouped as group III and Group IV where in group III include recent/ newly detected hypertension whose blood pressure value range _> 140/90 without medication (age range: 25-60 years) and group IV include individuals suffered from both hypertension and diabetes mellitus (FBS level of_>110mg\% with age range $25-60$ years. Fasting blood sugar was done by the glucose oxidaseperoxidase method (GOD-POD method). Results: hsCRP and plasma hemoglobin are associated with endothelial dysfunction and increases both with age as well as in disease condition. Endothelial dysfunction was demonstrated in asymptomatic young adults and children with risk factors for atherosclerosis as smoking cigarette and hypercholesterolemia. Conclusion: The presence of diseases such as hypertension or diabetes alone could affect increasing lipid profile value which may be strongly affected by increasing age. Therefore this study shows hsCRP and plasma hemoglobin associated with endothelial dysfunction and increase both with age as well as in disease condition with diabetes mellitus and hypertension together.
\end{abstract}

Keywords: Diabetes mellitus, Hypertension, hsCRP, CVD, Plasma hemoglobin.

\section{Introduction}

The process that importantly impact on their health status of elder people represents vascular aging. Aging process is one of the factors for main risk for the development of cardiovascular disease (CVD). Many studies show that $50 \%$ clinical CVD present in the elderly. Therefore complex structure and functional changes is associated with aging in vasculature independently of other risk factor as diabetes, hypertension and hypercholestesterolemia. ${ }^{[1]}$ Many studies epidemiologically show that risk factor for CVD is age. Actually the prevalence and incidence of coronary heart disease, hypertension, congestive heart failure and stroke steeply increase with age. ${ }^{[2]}$ Individuals in 75-84 year old as compared to 35-44 year old people increase in death rates for heart disease and cerebrovascular disease there is a $\sim 60$ fold and $\sim 80$ fold respectively. Cardiovascular aging is characterized by myocardial performance gradual deterioration of endothelial function in both experimental animals and humans. For Better understanding of the molecular mechanisms concealed aging process lead to pharmacological interventions that significantly delay age-related decay of cardiovascular function. ${ }^{[3,4,5,6,7,8]}$ Therefore strategies for the effect of aging in the vasculature could be potentially developed, alleviating CVD in the elderly population and the quality of life in the elderly population. ${ }^{[9]}$ The pathogenesis of many conditions like hypertension and atherosclerosis is key event to be proposed Dysfunction of the endothelium. Over the past decade, studies have assessed endothelial function in health and disease. ${ }^{[10]}$ The endothelium plays an active role in process of platelet adhesion and inflammation. C-reactive protein (CRP) is one of the acute phase proteins. Experimental and Laboratory shows that atherosclerosis in addition to being a disease of lipid accumulation, also represents a chronic inflammatory process. ${ }^{[11]}$ Traditional risk factors like smoking, age, obesity, hypertension, dyslipidemia and hyperglycemia may initiate endothelial damage. ${ }^{[12]}$ In 1992, endothelial dysfunction was demonstrated in asymptomatic young adults and children with risk factors for atherosclerosis as smoking cigarette and hypercholesterolemia. ${ }^{[13]}$

In the present study, is made to find out whether with increasing age develops endothelial dysfunction and also included subjects of younger age group and studied the changes take place with age as well as in disease state. Individuals who could develop diabetes 


\section{International Journal of Innovative Research in Medical Science (IJIRMS) \\ Volume 03 Issue 12 Dec 2018, ISSN: 2455-8737, Imp. Factor - 4.102 \\ Available online at $-\underline{w w w . i j i r m s . i n}$}

mellitus, hypertension heart related problems which are one of the major causes of morbidity and mortality in today's world.

The aim of study was screening of population with asymptomatic individuals for risk markers of hypertension and atherosclerosis leading to vascular events and also investigation of recently detected hypertension and diabetes mellitus.

\section{Materials and Methods:}

This study was carried out in the Dept. of Physiology at Zydus Medical College and hospital, Dahod .In these study total 200 indivuals were included. 2 control groups were include as 38 Group I healthy controls (Age range: 18-24 years) and 65 Group II controls included apparently healthy, asymptomatic individuals whose blood pressure values did not exceed 139/89 and whose fasting blood sugar (FBS) values were B110 mg\% (Age range: 2560 years). It is also grouped as group III and Group IV where in group III include recent/ newly detected hypertension whose blood pressure value range _> 140/90 without medication (age range: 2560 years) and group IV include individuals suffered from both hypertension and diabetes mellitus (FBS level of _>110mg\% with age range 25- 60 years. Individuals having uncontrolled diabetes or

complication due to extremely high sugar levels were not taken in this study. Height, weights and FBS as well as blood pressure of each individual were recorded. Blood pressures of every individual were recorded in a resting sitting position by standard sphygmomanometry.

Fasting blood sugar was done by the glucose oxidaseperoxidase method (GOD-POD method). Serum complete lipid profile that is; TC by enzymatic method. By using enzymatic method TG were calculated, by using the precipitation by phosphotungstate /manganese method high density lipoproteins (HDL) and Low density lipoproteins (LDL) and very low density lipoproteins (VLDL) were calculated using Friedewald's formula from TC, HDL-C and TG values. ${ }^{[13,14,15,16,17]}$ By using immuno turbidometric assay method serum hsCRP was estimated. By constructing a standard curve from the absorbance of the standards, hsCRP concentration of sample can be determined. ${ }^{[18]}$

Statistical analysis was carried out using unpaired ' $t$ ' test by using SPSS software. P value less than 0.001 (P\0.001) was considered as significant.

\section{Results and Observations:}

Table I: Age and Sex wise distribution of Patients:

\begin{tabular}{|l|c|c|c|c|}
\hline Study group & Group I & Group II & Group III & Group IV \\
\cline { 2 - 5 } & $\mathrm{N}=38$ & $\mathrm{~N}=65$ & $\mathrm{~N}=50$ & $25-60$ \\
\hline Age range (in years) & $18-14$ & $25-60$ & $25-60$ \\
\hline Sex Wise Distribution & 19 & 25 & 25 & 19 \\
\hline Male & 19 & 40 & 25 & 28 \\
\hline Female & \multicolumn{5}{|c|}{} \\
\hline
\end{tabular}

From the above table shows that total 200 individuals were include for this study which were which were distribution according to the age and sex. Total individuals were classified into 4 groups as group I, II, III and IV in which group I and II were compared as controls. In each group total no of individuals were also distributed in age as shown in above table.

Table II: Lipid profile of each subject was done and the results are summarized below

\begin{tabular}{|l|c|c|c|c|}
\hline Parameters & Group I & Group II & Group III & Group IV \\
\hline Serum TG (mg/dl) & $54.9-84.1$ & $78.6-130.6$ & $59.8-190.3$ & $95.2-170.3$ \\
\hline Range & $69.4 \pm 14.6$ & $110.8 \pm 41.7$ & $120.4 \pm 57.1$ & $137.8 \pm 51.5$ \\
\hline Serum TC (mg/dl) & $145.5-176.6$ & $152.8-242.4$ & $149.3-230.5$ & $169.6-229.8$ \\
\hline Range & $160.5 \pm 15.9$ & $198.5 \pm 34.8$ & $189.3 \pm 35.2$ & $199.2 \pm 30.7$ \\
\hline HDL-C (mg/dl) & $42.5-56.6$ & $33.3-51.6$ & $30.7-50.5$ & $36.2-52.4$ \\
\hline Range & $48.4 \pm 5.4$ & $42.3 \pm 8.7$ & $42.2 \pm 6.9$ & $44.1 \pm 8.1$ \\
\hline VLDL-C (mg/dl) & $11.0-16.8$ & $14.9-31.7$ & $11.2-38.8$ & $19.7-35.6$ \\
\hline Range & $13.9 \pm 2.9$ & $22.7 \pm 7.2$ & $25.1 \pm 13.3$ & $27.5 \pm 8.9$ \\
\hline LDL-C (mg/dl) & $82.6-111.2$ & $95.2-161.7$ & $88.4-160.4$ & $92.5-155.6$ \\
\hline Range & $95.6 \pm 13.2$ & $119.8 \pm 32.1$ & $113.2 \pm 35.2$ & $119.1 \pm 36.2$ \\
\hline
\end{tabular}

Table no II shows the level of lipid profile in all types of group. As comprised with group I and II in order to assess change in lipid profile levels. Group I recommended ranges of lipid profile with the references value where as group II,II and IV shows age group 25-60 years had higher lipid profile value with the reference range where as HDL-C was found to be lower of the reference range.

Table III: Other parameters like Hb, BMI, hsCRP and FBS of each subject was done and the results are summarized below

\begin{tabular}{|l|c|c|c|c|}
\hline Other Parameters & Group I & Group II & Group III & Group IV \\
\hline Plasma Hb (mg/dl) & $0.3-1.3$ & $0.5-4.7$ & $0.5-7.2$ & $0.8-7.4$ \\
\hline Range & $0.8 \pm 0.3$ & $1.7 \pm 0.8$ & $3.4 \pm 1.6$ & $3.3 \pm 1.5$ \\
\hline BMI(Kg/M2) & $16-25$ & $22-28$ & $21-28$ & $23-30$ \\
\hline Range & $20 \pm 4$ & $24 \pm 2$ & $24 \pm 3$ & $26 \pm 2$ \\
\hline hsCRP(mg/L) & $0.2-1.4$ & $0.4-3.5$ & $0.7-5.8$ & $0.8-5.4$ \\
\hline Range & $0.6 \pm 0.4$ & $1.76 \pm 0.83$ & $3.10 \pm 1.38$ & $3.15 \pm 1.30$ \\
\hline FBS (mg/d) & $84.6-97.6$ & $87.7-99.7$ & $89.3-102.2$ & $114.9-200.6$ \\
\hline Range & $91.6 \pm 7.0$ & $94.2 \pm 6.8$ & $97.7 \pm 8.5$ & $150.0 \pm 25.1$ \\
\hline
\end{tabular}




\section{International Journal of Innovative Research in Medical Science (IJIRMS) Volume 03 Issue 12 Dec 2018, ISSN: 2455-8737, Imp. Factor - 4.102 \\ Available online at $-\underline{w w w . i j i r m s . i n}$}

In above table no III group II shows higher age group control have hsCRP value in normal range whereas higher as compared with lower age group ( group I) control which suggested that age itself is a major risk factor to most health problems. Individuals in group III suffered from hypertension showed higher value of hSCRP as compare with group II age controls. Hemoglobinemia means increased hemolysis is now considered as a novel mechanism of human disease. Above table shows that significantly high levels of hemoglobin in plasma is not only in diabetic hypertensives and hypertensives but also in middle aged controls.

\section{Discussion and Conclusion:}

Nowadays hemoglobinemia which means increasing hemolysis is consider as a novel mechanism of human disease. During intravascular hemolysis, hemoglobin is released into plasma from erythrocytes. Significantly high levels of hemoglobin in plasma were seen in this study not only in diabetic hypertensives and hypertensives but also in middle aged controls. In this study, Group II higher age group controls have hsCRP values within the normal range and have higher as compared to the lower age group (Group I) controls which suggest that for most health problems major risk factor is age itself. As compared to the Group II age-matched controls Group III patients who suffered from hypertension showed significantly high values of hsCRP. There are increased CRP values for Type 2 diabetic patients. In group IV there were significantly higher hsCRP values patients who suffered from both diabetes mellitus and hypertension as compared to Group II agematched controls. There were also higher hsCRP values in group IV than patients who suffered from only hypertension in group III.

TC, TG, high-density lipoprotein cholesterol (HDL-C), lowdensity lipoprotein cholesterol (LDL-C) and very low density lipoprotein cholesterol (VLDL-C) were also done as shown in table above.

Group I shows well within the recommended ranges of lipid profile values of which TC is $150-250 \mathrm{mg} \%$, TG is $50-150 \mathrm{mg} \%$, and HDL-C is $30-70 \mathrm{mg} \%$ whereas LDL-C is $90-160 \mathrm{mg} \%$ and VLDL-C is $10-30 \mathrm{mg} \%$ as reference value. ${ }^{[13,19 \& 17]}$ Groups II, III and IV of the age group 25-60 years, had lipid profile values on the higher side of the reference ranges and HDLC was found to be on the lower side of the reference range.

Studies shows that a relationship between type 2 diabetes mellitus and hypertension metabolic abnormalities and several vascular. ${ }^{[20]}$ For impairment of EDRF (NO) function increased plasma hemoglobin levels are responsible which may be the causes of disease process. Exact mechanism of inactivating EDRF (NO) by hemoglobin is not known. ${ }^{[21]}$ Therefore aging process is noted as a part of released more hemoglobin in plasma. At study of Atre et al shows that patients suffering from hypertension reported high values of plasma hemoglobin as compared to normal controls. ${ }^{[22]}$ Endothelial dysfunction is also related to CRP. ${ }^{[23]}$ According to the study of Saito et al shows that variations in circulating hsCRP even in normal range is involved in the interrelation of cardiovascular risk factors like age, obesity, smoking, blood pressure and dyslipidemia that support for promotion to atherosclerosis and ultimately provoke cardiovascular diseases, such as coronary artery disease. ${ }^{[24]}$ Other studied by Lima et al observed higher levels of hsCRP than normal indivuals who suffered from both diabetes and hypertension which indicating individuals with two associated diseases have a more active inflammatory state. ${ }^{[25]}$

However the presence of diseases such as hypertension or diabetes alone could affect increasing lipid profile value which may be strongly affected by increasing age. Therefore this study shows
hsCRP and plasma hemoglobin associated with endothelial dysfunction and increase both with age as well as in disease condition with diabetes mellitus and hypertension together.

\section{References}

[1] Barodka, V. M., Joshi, B. L., Berkowitz, D. E.,Hogue,C.W. Jr., and Nyhan,D. (2011). Review article: implications of vascular aging. Anesth. Analg. 112, 1048-1060.

[2] Samer S. Najjar; Angelo Scuteri; Edward G. Lakatta Arterial Aging. Is It an Immutable Cardiovascular Risk Factor? Hypertension. 2005;46:454- 462

[3] Yang B, Larson DF, Watson RR. Life Sci 2004;75:65567.

[4] Lakatta EG. Circulation 2003;107:490-7.

[5] Lakatta EG, Levy D. Circulation 2003;107:346-54.

[6] Lakatta EG, Levy D. Circulation 2003;107:139-46.

[7] Sussman MA, Anversa P. Annu Rev Physiol 2004;66:29-48.

[8] Csiszar A, Pacher P, Kaley G, Ungvari Z. Curr Vasc Pharmacol 2005;3:285-91.

[9] Najjar, S. S., Scuteri, A., and Lakatta, E. G. (2005). Arterial aging: is it an immutable cardiovascular risk factor? Hypertension 46, 454-462.

[10] Moreno H Jr, Chalon S, Urae A, Tangphao O, Abiose $\mathrm{AK}$, Hoffman BB, et al. Endothelial dysfunction in human hand veins is rapidly reversible after smoking cessation. Am J Physiol Heart Circ Physiol. 1998;275:H1040-5

[11] Ross R. Atherosclerosis: an inflammatory disease. N Engl J Med. 1999;340:115-26

[12] Libby P, Ridker OM, Maseri A. Inflammation and atherosclerosis. Circulation. 2002;105:1135-43.

[13] Allain CC, Poon LS, Chan CS, Richard W, Fu PC. Enzymatic determination of total serum cholesterol. Clin Chem. 1974;20(4): 470-5.

[14] Varley H, Gowenlock AH, McMurray JR, McLauchlan DM, editors. Varley's practical clinical biochemistry. 6th ed. London: Heinemann Medical Books; 1988. p. 322-5.

[15] Tietz NW. Clinical guide to laboratory tests. 2nd ed. Philadelphia: WB Saunders; 1994. p. 1073-91.

[16] Warnik GR, Benderson J, Albers JJ. Dextran sulfate$\mathrm{Mg}$ ?? Precipitation procedure for quantitation of high density lipoprotein cholesterol. Clin Chem. 1982;28(6):1379-88.

[17] Friedewald WT, Levy RI, Fredrickson DS. Estimation of the concentration of low density lipoprotein cholesterol in plasma, without use of the preparative ultracentrifuge. Clin Chem. 1972;18: 499-502.

[18] Kindmark CO. The concentration of CRP in sera from healthy individuals. Scand J Clin Lab Invest. 1972;229:407-11.

[19] Tietz NW. Clinical guide to laboratory tests. 2nd ed. Philadelphia: WB Saunders; 1994. p. 1073-91. 
[20] Ganne S, Arora SK, Dotsenko O, McFarlane SI, WhaleyConnell A. Hypertension in people with diabetes and the metabolic syndrome: patho-physiologic insights and therapeutic update. Curr Diab Rep. 2007;7(3):208-17.

[21] Vane JR, A* nggard EE, Botting RM. Regulatory functions of the vascular endothelium. $\mathrm{N}$ Engl J Med. 1990;323:27-36.

[22] Atre AL, Patki PS, Nandal DH, Yegnanarayan R. Plasma hemoglobin: a novel biochemical marker in hypertension, congestive cardiac failure and myocardial infarction. Med J West India. 1999;27:28-32.

[23] Fichtlscherer S, Rosenberger G, Walter D, Breuer S, Dimmeler S, Zeiher A. Elevated C-reactive protein levels and impaired endothelial vasoreactivity in patients with coronary artery disease. Circulation. 2000;102:1000-6.

[24] Saito M, Ishimitsu T, Minami J, Ono H, Ohrui M, Matsuoka H. Relations of plasma high-sensitivity Creactive protein to traditional cardiovascular risk factors. Atherosclerosis. 2003;167(1): 73-9.

[25] Lima LM, Carvalho MG, Soares AL, Sabino Ade P, Fernandes AP, Novelli BA, et al. High-sensitivity Creactive protein in subjects with type 2 diabetes mellitus and/or high blood pressure. Arq Bras Endocrinol Metab. 2007;51(6):956-60. 